measurable sets in such a way that the indefinite integrals (in the $D-K-Y$ sense) are absolutely continuous on each of these, ${ }^{*}$ then the conditions of Theorem 9 are satisfied when $J(a, b)$ denotes the $D-K-Y$ integral of $f(x), E$ is any everywhere dense set on $X, M$ is $X_{0}$, and $F(x) \equiv f(x)$ on $X_{0}$.

It is a very simple matter to extend the results of the present paper to cases where the interval of definition $X$ is replaced by any measurable point set $E$ on $X$. The definition of $f(x)$ is extended to points of $X-E$ by letting $f(x)$ be zero at such points. The integral of $f(x)$ on $E$ is then described in terms of the integral of the extended function on $X$. One could define a simple function on a point set by saying that it is a function which takes on only a finite number of values on this set. This definition is not needed in the present adaptation.

The University of California at Los Angeles

\title{
REFLECTIONS IN FUNCTION SPACE $\dagger$
}

BY L. S. KENNISON

1. Introduction. The purpose of this paper is to point out an error, $\ddagger$ giving the corrected form of the incorrect theorem referred to below as Delsarte's theorem, also to prove a generalization. However, the contribution made to the geometry of function space may be interesting to some on its own account.

We shall consider only functions of one or two variables defined on the interval $(a, b)$ or the corresponding square. All such functions are to be bounded and integrable on the range of definition. We shall denote the continuous arguments on $(a, b)$ by the letters $x, s, t, u$, written as subscripts or superscripts and shall imply integration on $(a, b)$ with respect to any argument that occurs twice in the same term.

\footnotetext{
* See Khintchine, Comptes Rendus, vol. 152 (1916), p. 290.

$\dagger$ Presented to the Society, February 28, 1931.

$\ddagger$ See $\$ 3$ below.
} 
2. Functional Rotations and Reflections. Delsarte* has defined functional rotations as follows. The functional transformation $\dagger$

$$
y^{x}=\bar{y}^{x}+K_{s}^{x} \bar{y}^{s},
$$

from $\bar{y}^{x}$ to $y^{x}$, is a functional rotation if and only if

$$
\begin{aligned}
& K_{s}^{x}+K_{x}^{s}+K_{x}^{u} K_{s}^{u}=0, \\
& K_{s}^{x}+K_{x}^{s}+K_{u}^{x} K_{u}^{s}=0 .
\end{aligned}
$$

Delsarte shows that these two conditions are equivalent. A kernel such that these are satisfied is called a kernel of rotation. From Fredholm's product theorem we see that

$$
D_{k}(-1)= \pm 1
$$

where $D_{k}(\lambda)$ is defined in the usual manner. Delsarte $\ddagger$ states and attempts to prove the following theorem, which we shall call Delsarte's Theorem.

The necessary and sufficient condition that a kernel be one of rotation is that the kernel be the value taken by the resolvent of a skewsymmetric kernel for the value $\lambda=1 / 2$ of the parameter.

Let $f^{x}=f_{x}$ be a function such that $f^{x} f_{x}=1$, and consider the functional transformation

$$
\bar{y}^{x}=y^{x}-2 f^{x} f_{s} y^{s} .
$$

Denote by $g_{1}$ the Fourier coefficient of an arbitrary function $g^{x}$ with respect to the set of one normal and orthogonal function $f^{x}$. Then we may write

$$
g^{x}=g_{1} f^{x}+r^{x},
$$

where $r^{s} f_{s}=0$. Applying (5), we have

$$
\bar{g}^{x}=-g_{1} f^{x}+r^{x} .
$$

Hence the transformation (5) reverses the component of $g^{x}$ in the direction of $f^{x}$ and leaves unchanged the component $r^{x}$ orthogonal to $f^{x}$. In other words, the function $\bar{g}^{x}$ may be thought of

* Annales de Toulouse, vol. 20 (1928), p. 47 ff.

$\dagger$ The corresponding integral equation, in Delsarte's notation, is of the form $\bar{y}(x)=y(x)+\lambda \int_{a}^{b} K(x, s) \tilde{y}(s) d s$, which reduces to (1) for $\lambda=-1$.

$\ddagger$ Loc. cit., p. 56 . 
as the reflection of $g^{x}$ in the ( $\left.\infty-1\right)$-dimensional variety orthogonal to $f^{x}$ and containing the origin of function space. Accordingly we call (5) a functional reflection.

3. Proper and Improper Rotations. Now call any kernel of rotation that is the resolvent of a skew-symmetric kernel with parameter $\frac{1}{2}$ a proper kernel of rotation, and the corresponding transformation (1) a proper functional rotation. Any other kernel of rotation will be called an improper kernel of rotation, and its transformation an improper functional rotation. Delsarte's theorem states that every kernel of rotation is proper.

Theorem 1. Every functional reflection is an improper functional rotation.

It is easy to verify that (2) and (3) are satisfied for the kernel $-2 f^{x} f_{s}$. It is also seen by a little calculation that the resolvent of a skew-symmetric kernel cannot be symmetric for any value of the parameter. Hence $-2 f^{x} f_{s}$ is an improper kernel of rotation. This contradiction with Delsarte's theorem is explained by the following mistake in Delsarte's proof. On page 55 (loc. cit.) Delsarte defines the kernel $h(s, t)$ as $\Gamma(s, t ;-1 / 2)$, where $\Gamma(s, t ; \lambda)$ is the resolvent kernel of $K(s, t)$, the kernel of rotation in question. He then proves that $h(s, t)$ is skew-symmetric and that $K(s, t)$ is the value of the resolvent of $h(s, t)$ for $\lambda=1 / 2$. If $-1 / 2$ is a pole of $\Gamma(s, t ; \lambda)$, then $h(s, t)$ is not defined, and Delsarte's theorem fails to hold. It is readily seen that the resolvent of the kernel $-2 f^{x} f_{s}$ is

$$
-\frac{2 f^{x} f_{s}}{1+2 \lambda}
$$

for which $\lambda=-1 / 2$ is a pole. With this mistake corrected, Delsarte's theorem takes the following form:

THEOREM 2. A kernel of rotation, for which $-1 / 2$ is not a pole of the resolvent, is a proper kernel of rotation.

The generalization of Theorem 2 is as follows.

THEOREM 3. Let $K_{s}^{x}$ be a kernel of rotation, such that $-1 / 2$ is an $n$-fold root of its Fredholm determinant $D(\lambda)$. Then the functional transformation (1) corresponding to $K_{s}^{x}$ is the product of $n$ functional reflections followed by a proper functional rotation. 
LEMMA 1. Let $K_{s}^{x}$ be a kernel of rotation, such that $-1 / 2$ is an $r$-fold root of its Fredholm determinant. Then the functional transformation (1) corresponding to $K_{s}^{x}$ is the product of a functional reflection and a rotation for whose kernel $-1 / 2$ is an $(r-1)$-fold root of the Fredholm determinant.

Let $\phi^{x}$ be a normalized fundamental function of $K_{s}^{x}$, corresponding to $\lambda=-1 / 2$. Consider the kernel $L_{s}^{x}=-2 \phi^{x} \phi^{s}$ and $R_{s}^{x}=K_{s}^{x}-L_{s}^{x}$. Then $K_{s}^{x} \phi^{s}=-2 \phi^{x}$ and we have

$$
L_{s}^{x}+R_{s}^{x}+R_{t}^{x} L_{s}^{t}=K_{s}^{x},
$$

and so $D_{K}(\lambda)=(1+2 \lambda) D_{R}(\lambda)$.

Direct substitution of $K_{s}^{x}+2 \phi^{x} \phi_{s}$ in (3) shows that (3) is satisfied for $R_{s}^{x}$. Multiplying (2) by $\phi^{s}$, integrating, and using $K_{s}^{x} \phi^{s}=-2 \phi^{x}$, we obtain $K_{s}^{x} \phi_{x}=-2 \phi_{s}$, which is sufficient to show that (2) is satisfied for $R_{s}^{x}$. Hence $R_{s}^{x}$ is a kernel of rotation and the lemma is proved. Applying the lemma $n$ times, we obtain the theorem.

The formula

$$
D_{K}(\lambda)=(1+2 \lambda)^{n} D_{P}(\lambda)
$$

is also obtained by a repetition of the lemma, where $D_{P}(\lambda)$ is the determinant of the proper kernel of rotation, $P_{s}^{x}$, of Theorem 3.

LEMma 2. The Fredholm determinant $D(-1)$ of a proper functional rotation is +1 .

Let $P_{s}^{x}$ be a proper kernel of rotation. Then, by Theorem 2, there exists a skew-symmetric kernel $H_{s}^{x}$ of which $P_{s}^{x}$ is the resolvent for $\lambda=1 / 2$. The Fredholm determinant of $H_{s}^{x}$ is positive for all real $\lambda$, since it is +1 for $\lambda=0$, is continuous in $\lambda$, and has no real roots. Also $D_{H}(1 / 2) D_{P}(-1)=1$; hence $D_{P}(-1)$ is positive, and by (4) is +1 . Setting $\lambda=-1$ in (6), we find the following theorem.

THEOREM 4. The determinant $D_{K}(-1)$ of the transformation $(1)$, is equal to $(-1)^{n}$, where $n$ is the multiplicity of the root $-1 / 2$ of $D_{K}(\lambda)$.

Princeton University 